\title{
Response to the Letter to the Editor Surgery for high-grade unruptured arteriovenous malformations: era for a new paradox? by Bervini and Morgan
}

\author{
Hans-Jakob Steiger $^{1} \cdot$ Igor Fischer $^{2} \cdot$ Benjamin Rohn $^{1} \cdot$ Bernd Turowski $^{3}$. \\ Nima Etminan ${ }^{1} \cdot$ Daniel Hänggi $^{1}$
}

Received: 11 August 2015 / Accepted: 12 August 2015 /Published online: 23 August 2015

(C) Springer-Verlag Wien 2015

We appreciate the comments by Bervini and Morgan regarding our publication "Microsurgical resection of Spetzler-Martin grades 1 and 2 unruptured brain arteriovenous malformations results in lower long-term morbidity and loss of quality-adjusted life-years (QALY) than conservative management- Results of a single group series". They challenge our somewhat-simplified conclusion that at present a benefit of microsurgical treatment of Spetzler-Martin (SM) grade 1 and 2 arteriovenous malformation (AVMs) can be accepted with certainty while this is not the case for SM grades 3 and 4. They argue that, despite its high morbidity, microsurgical treat-

Hans-Jakob Steiger

steiger@uni-duesseldorf.de

Department of Neurosurgery, Heinrich-Heine-Universität, Düsseldorf, Germany

2 Division of Informatics and Statistics, Department of Neurosurgery, Heinrich-Heine-Universität, Düsseldorf, Germany

3 Division of Neuroradiology, Institute of Radiology, Heinrich-Heine-Universität, Düsseldorf, Germany ment mortality for unruptured SM grade 3 AVM is likely lower than the long-term mortality resulting from the natural history.

In general, it is somewhat difficult to compare a onetime risk (i.e., treatment-related morbidity) with a longterm risk (natural history of AVMs) as these are two different quantities: Is it better for a 40 -year-old patient to live with a permanent neurological deficit without the threat of hemorrhage and premature death, or to live and work normally up to the age of 50 or 60 and then possibly suffer a hemorrhage and die suddenly? We tried to partially solve this dilemma by normalizing morbidity and mortality in terms of lost qualityadjusted life-years (QALY). Although with this approach SM grade 3 as a whole group did not benefit from microsurgery, patients younger than 39 years did. A clear benefit of surgical treatment was seen in the younger patients with SM grade 3 AVMs. The surgical risk in these patients with respect to lost QALY was negated by the natural course within 5-7 years. However, statistical uncertainty due to the small sample size precluded us from drawing more definitive conclusions regarding the potential benefit of microsurgical resection in younger patients with SM grade 3 AVM. Thus, we agree that the appropriate management of AVM patients requires careful and nuanced assessment of risks and benefits of both invasive and conservative treatment for every patient individually until more data in this respect become available. 